\title{
EVALUATION OF FAT-FREE MASS BY WHOLE-BODY COUNTER IN JAPANESE HEALTHY YOUNG ADULTS
}

\author{
Naoko Morita ${ }^{1}$, Noboru Takamura ${ }^{2, *}$, Tomohiko Murakami ${ }^{2}$, Ohzora $\mathrm{Jo}^{2}$, Kiyoshi Aoyagi ${ }^{2}$, \\ Shunichi Yamashita ${ }^{3,4}$ and Yutaka Okumura ${ }^{1}$ \\ ${ }^{1}$ Department of Radiation Biophysics, Nagasaki University Graduate School of Biomedical Sciences, \\ Nagasaki University, Nagasaki, Japan \\ ${ }^{2}$ Department of Public Health, Nagasaki University Graduate School of Biomedical Sciences, Nagasaki \\ University, Nagasaki, Japan \\ ${ }^{3}$ Department of Molecular Medicine, Nagasaki University Graduate School of Biomedical Sciences, \\ Nagasaki University, Nagasaki, Japan \\ ${ }^{4}$ Department of Protection of the Human Environment, World Health Organization, Geneva, \\ Switzerland
}

Received February 10 2006, amended March 24 2006, accepted May 292006

\begin{abstract}
Whole-body counters (WBCs) are special instruments for measuring internal irradiation doses and are usually housed within or around nuclear facilities in the event of unexpected radiation emergencies. As a substantial proportion of total body potassium (TBK) is found in fat-free mass (FFM), FFM volume can be predicted from WBC-measured ${ }^{40} \mathrm{~K}$. We screened TBK in Japanese healthy young adults using a WBC and found strong linear correlations between TBK and lean body mass (LBM) and body mass index $(r=0.97, P<0.01$ and $r=0.47, P<0.01$, respectively). Multiple linear regression analysis, following adjustments for sex, indicates that only LBM has a significant correlation with TBK $(P<0.01)$. These results strongly support the feasibility of using WBCs for estimating FFM.
\end{abstract}

\section{INTRODUCTION}

Whole-body counters (WBCs) are used for measuring internal radiation exposure. In preparation for unexpected radiation emergencies, WBCs are typically housed close to nuclear facilities such as nuclear power plants. WBCs are already housed in all Japanese nuclear facilities. As radiation emergencies are rare, the development of alternative applications for WBCs would provide important regular training for WBC operators as well as maintenance of the machines.

Fat-free mass (FFM) represents the largest tissue component of the human body. Whole-body FFM is influenced by several factors, including age, sex, race, physical activity and disease state ${ }^{(1)}$. Objective quantification of FFM mass is important in the field of clinical physiology.

At present, the most accurate in vivo methods of evaluating whole-body FFM are multislice magnetic resonance imaging and computed tomography $(\mathrm{CT})^{(2,3)}$; however, as CT exposes subjects to radiation, its use should be carefully considered. Potassium is measurable in vivo ${ }^{(4)}$. Since body potassium exists throughout the FFM compartment, a large proportion of the total body potassium (TBK) exists in FFM. This suggests that the ratio of TBK to FFM is relatively stable in healthy adults, providing

*Corresponding author: takamura@net.nagasaki-u.ac.jp a method of predicting FFM by evaluating the volume of potassium in the body. Since potassium constantly contains $0.0117 \%$ of radioactive potassium $\left({ }^{40} \mathrm{~K}, T_{1 / 2}=1.28 \times 10^{9} \mathrm{y}\right)$, measuring levels of ${ }^{40} \mathrm{~K}$ using a WBC should provide an accurate method for evaluating FFM volume with high sensitivity, low cost and no radiation exposure ${ }^{(5)}$.

In this study, we evaluate the suitability of WBCs for the measurement of FFM to develop new opportunities for WBC use at nuclear facilities.

\section{MATERIALS AND METHODS}

\section{STUDY PARTICIPANTS AND BODY COMPOSITION MEASUREMENTS}

We screened 54 Japanese healthy young adults (21 men: mean age $21.7 \pm 1.2$ y; 33 women: mean age $21.3 \pm 2.8$ y). Participants' heights were measured using a height scale (AD-6227, AND, Tokyo). According to manufacturer's instruction, total body mass and fat mass percentage ( $\%$ fat) were measured by bioelectrical impedance analysis using a tetrapolar body-fat analyser (TBF-401, TANITA, Tokyo), with participants wearing only their underwear during analysis.

Body mass index (BMI, $\mathrm{kg} \mathrm{m}^{-2}$ ) is defined as subject weight $(\mathrm{kg})$ divided by the square of subject height $(\mathrm{m})$, while lean body mass (LBM, kg) is derived by subtracting fat mass from the total body mass. 


\section{Measurement of total body POTASSIUM}

The WBC (Fuji Electric Holdings Co., Ltd, Tokyo, Japan) system at the Atomic Bomb Disease Institute, Nagasaki University Graduate School of Biomedical Sciences, was used for ${ }^{40} \mathrm{~K}$ measurement. The principle of whole-body measurement has been described previously ${ }^{(6)}$. Briefly, WBC consists of two $\mathrm{NaI}(\mathrm{Tl})$ scintillators (upper and lower) each connected to four photomultiplier (PM) tubes through a light guide. The detectors and bed are mounted in an iron room, with walls, roofs and floor made of pre-World War II steel $20 \mathrm{~cm}$ in thickness. The shelter is lined with $3 \mathrm{~mm}$ of lead on the inside to reduce background count in the low-energy region. The air in the steel room is filtered and the air pressure in the steel room is maintained higher than that in the surrounding laboratory, which in turn has a slightly higher air pressure than the outside. Whole-body counting was performed on a stretcher. Scintillators moved longitudinally along the middle of the body with a constant speed $\left(10 \mathrm{~cm} \mathrm{~min}{ }^{-1}\right)$, in order to screen a whole body with a uniform sensitivity. Collimation was not used for the current study. The gamma spectrum was recorded using a multichannel analyser connected on-line to a computer. The multichannel analyser was calibrated once a month by potassium chloride. The individual data were analysed using WinEMCA version 1.00 (Seiko EG\&G Co., Ltd, Tokyo).

${ }^{40} \mathrm{~K}$ peak counts were recognised within the range of $1.35-1.57 \mathrm{MeV}$, and the TBK detection limit was $1.45 \mathrm{~g}$, which was calculated according to operating instruction of WBC. The counting efficiency of ${ }^{40} \mathrm{~K}$ was $3.10 \times 10^{-1}$.

The calculation factor was derived using an anthropometric phantom, which was filled with a potassium chloride solution ${ }^{(7)}$ and was determined in each subject according to chest thickness and body weight. Obtained values were corrected by background radiation obtained before the measurement, and TBK was calculated according to the WBC operating instructions using the following formula: TBK $(\mathrm{g})={ }^{40} \mathrm{~K}$ (counts $\mathrm{m}^{-1}$ )/calculation factor (counts $\mathrm{m}^{-1} \mathrm{~g}^{-1}$ ).

We performed Pearson correlation analysis and multiple regression analysis to evaluate the correlations between TBK and BMI, and TBK and LBM. All statistical analyses were performed by SPSS14.0 ${ }^{\circledR}$ (SPSS Japan Inc., Tokyo, Japan), according to its instruction. A probability value $<0.05$ was considered to indicate significance.

\section{RESULTS AND DISCUSSION}

We observed a weak correlation between TBK and BMI $(r=0.46, P<0.01)$, although a relatively strong correlation was observed when data were separated by sex (men, $r=0.80$; women, $r=0.72$; $P<0.01$ for both). In contrast, a strong linear correlation was observed between TBK and LBM $(r=0.97, P<0.01$; Figure 1). Multiple linear regression analysis following adjustment for sex indicated that only LBM had a significant correlation with TBK $(P<0.01$; Table 1$)$.

The current results suggest that ${ }^{40} \mathrm{~K}$ measurement by WBC enables the estimation of FFM with high sensitivity, low cost and no radiation exposure. Whole-body counting is a sensitive method for evaluating internal irradiation such as the ${ }^{137} \mathrm{Cs}$ burden in residents surrounding the Chernobyl Nuclear Power Plant ${ }^{(8)}$.

Objective quantification of SM mass is important in the field of clinical physiology. Furthermore, since CT exposes subjects to unnecessary ionising radiation, its use for research purposes is not usually approved by institutional review boards, particularly in studies of healthy subjects.

Measurement of TBK by WBC is rapid, convenient and simple to perform. This method can be applied to subjects of any age because no radiation exposure occurs and active participation of the subject is not necessary. From this point of view,

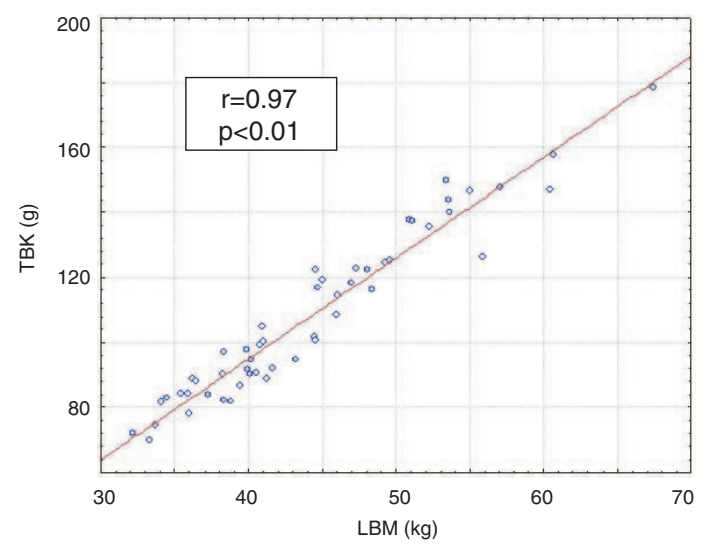

Figure 1. Correlation between LBM and TBK.

Table 1. Multiple linear regression analysis for TBK adjusted for sex.

\begin{tabular}{lllll}
\hline Variables & \multicolumn{1}{c}{ SE } & \multicolumn{1}{c}{$95 \% \mathrm{CI}$} & \multicolumn{1}{c}{$P$} \\
\hline BMI & 0.44 & 0.49 & $-0.55,1.4$ & 0.38 \\
LBM & 2.3 & 0.24 & $1.86,2.8$ & $<0.01$ \\
\hline
\end{tabular}

The regression coefficient $(\beta)$ is the average amount the dependent increases when the variable increases one unit and other variables are held constant. 95\% confidence interval (CI) represents the plus/minus range around the observed sample regression coefficient. If the CI includes 0 , then there is no significant linear relationship 


\section{N. MORITA ET AL.}

WBC can be applied in sports science such as the evaluation of physical training effects through the periodical quantification of FFM. Furthermore, TBK measurements can also be used to estimate body cell mass, an important body component at the cellular level ${ }^{(9)}$.

The current study did not include any children or patients with underlying diseases. Further studies are needed to clarify the utility of WBCs in different populations and to develop other possible conventional uses of this equipment for times other than periods of radiation emergency.

\section{REFERENCES}

1. Forbes, G. B. Perspectives on body composition. Curr. Opin. Clin. Nutr. Metab. Care 5, 25-30 (2002).

2. Lukaski, H. C. Estimation of muscle mass. In Human Body Composition. Roche, A. F., Heymsfield, S. B. and Lohman, T. G., Eds. (Champaign, IL: Human Kinetics Publishers) pp. 109-128 (1996).

3. Janssen, I., Heymsfield, S. B., Baumgartner, R. N. and Ross, R. Estimation of skeletal muscle mass by bioelectrical impedance analysis. J. Appl. Physiol. 89, 465-471 (2000).

4. Snyder, W. S., Cook, M. J., Nasset, E. S., Karhausen, L. R., Howells, G. P. and Tipton, I. H. Report of the Group on Reference Man (Oxford: Pergamon Press) pp. 312-313 (1975).

5. Wang, Z., Zhu, S., Wang, J., Pierson, R. N. and Heymfield, S. B. Whole-body skeletal muscle mass: development and validation of total-body potassium prediction models. Am. J. Clin. Nutr. 77, 76-82 (2003).

6. Morita, N., Takamura, N., Ashizawa, K., Shimasaki, T., Yamashita, S. and Okumura, Y. Measurement of whole body Cs-137 in residents around Chernobyl Nuclear Power Plant. Radiat. Prot. Dosim. 113, 326-329 (2005)

7. Sousa Wde, O., Dantas, B. M. and Lipsztein, J. L. In vivo measurement technique of ${ }^{40} \mathrm{~K}$ as an indicator of the amount of skeletal muscle tissue in the human body. Radiat. Prot. Dosim. 105, 487-490 (2003).

8. Yamashita, S. and Shibata, Y., Eds. Chernobyl: $A$ Decade (Amsterdam: Elsevier), ICS 1156, 613 pp. (1997).

9. Moore, F. D. and Boyden, C. M. Body cell mass and limits of hydration of the fat-free body: their relation to estimated skeletal weight. Ann. NY Acad. Sci. 110, 62-71 (1963). 